\title{
Correlation between compliance and brace treatment in juvenile and adolescent idiopathic scoliosis: SOSORT 2014 award winner
}

\author{
Angelo G Aulisa ${ }^{1 *}$, Marco Giordano ${ }^{1}$, Francesco Falciglia', Emanuele Marzetti ${ }^{2}$, Andrea Poscia ${ }^{3}$ and Vincenzo Guzzanti ${ }^{1,4}$
}

\begin{abstract}
Background: Over the last years, evidence has accumulated in support of bracing as an effective treatment option in patients with idiopathic scoliosis. Yet, little information is available on the impact of compliance on the outcome of conservative treatment in scoliotic subjects. The aim of the present study was to prospectively evaluate the association between compliance to brace treatment and the progression of scoliotic curve in patients with idiopathic adolescent (AIS) or juvenile scoliosis (JIS).

Methods: Among 1.424 patients treated for idiopathic scoliosis, 645 were eligible for inclusion criteria. Three outcomes were distinguished in agreement with the SRS criteria: curve correction, curve stabilization and curve progression. Brace wearing was assessed by one orthopaedic surgeon (LA) and scored on a standardized form. Compliance to treatment was categorized as complete (brace worn as prescribed), incomplete A (brace removed for 1 month), incomplete B (brace removed for 2 months), incomplete C (brace removed during school hours), and incomplete D (brace worn overnight only). Chi square test, T test or ANOVA and ANOVA for repeated measures tests were used as statistical tests.
\end{abstract}

Results: The results from our study showed that at follow-up the compliance was: Complete 61.1\%; Incomplete A 5.2\%; Incomplete B 10.7\%; Incomplete C 14.2\%; Incomplete D 8.8\%. Curve correction was accomplished in 301/319 of Complete, 19/27 Incomplete A, 25/56 Incomplete B, 52/74 Incomplete C, 27/46 Incomplete D. Cobb mean value was 29.8 $\pm 7.5 \mathrm{SD}$ at beginning and 17.1 $\pm 10.9 \mathrm{SD}$ at follow-up. Both Cobb and Perdriolle degree amelioration was significantly higher in patients with complete compliance over all other groups, both in juvenile, both in adolescent scoliosis. In the intention-to-treat analysis, the rate of surgical treatment was $2.1 \%$ among patients with definite outcome and $12.1 \%$ among those with drop-out. Treatment compliance showed significant interactions with time.

Conclusion: Curve progression and referral to surgery are lower in patients with high brace compliance. Bracing discontinuation up to 1 month does not impact on the treatment outcome. Conversely, wearing the brace only overnight is associated with a high rate of curve progression.

Keywords: Compliance, Juvenile idiopathic scoliosis, Adolescent idiopathic scoliosis, PASB brace, Conservative treatment

\section{Background}

Idiopathic scoliosis is a 3-dimensional spine deformity affecting $0.3-0.5 \%$ of children younger than 16 years of age $[1,2]$. Bracing is frequently prescribed as a non-surgical treatment option to patients with idiopathic scoliosis and a spinal curvature greater than $20^{\circ} \mathrm{Cobb}$. Notably, bracing

\footnotetext{
* Correspondence: angelogabriele.aulisa@fastwebnet.it

'U.O.C. of Orthopedics and Traumatology, Children's Hospital Bambino Gesù, Institute of Scientific Research, P.zza S. Onofrio, Rome 4-00165, Italy

Full list of author information is available at the end of the article
}

is the only conservative approach with proven effectiveness in such condition [3-8]. Several factors may have influence the efficacy of brace treatment, including age, gender, bone maturity, prescribed hours of bracing, and curve pattern and magnitude. In particular, a recent Cochrane Review [9-11] and a meta-analysis by Rowe [12] have shown that compliance has a great impact on the outcome of bracing. Indeed, the guidelines released by the Society on Scoliosis Orthopedic and Rehabilitation

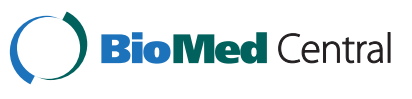

(c) 2014 Aulisa et al.; licensee BioMed Central Ltd. This is an Open Access article distributed under the terms of the Creative Commons Attribution License (http://creativecommons.org/licenses/by/4.0), which permits unrestricted use, distribution, and reproduction in any medium, provided the original work is properly credited. The Creative Commons Public Domain Dedication waiver (http://creativecommons.org/publicdomain/zero/1.0/) applies to the data made available in this article, unless otherwise stated. 
Treatment (SOSORT) indicate adherence as a key element in determining the efficacy of bracing [13,14].

Given these considerations, the evaluation of compliance to bracing through the use of either dedicated questionnaires [15-18] or the application of sensors within the brace [13,19-25], increases the internal validity of clinical investigations on the topic. The aim of the present study was to prospectively evaluate the association between compliance to bracing and the progression of scoliotic curve, including surgery referral rate, in patients with idiopathic adolescent and juvenile scoliosis.

\section{Methods \\ Study design and participants Patients}

This is a prospective study based on ongoing database including 1,424 patients treated for idiopathic scoliosis. Of these 522 patients whit a definite outcome were included, while 120 were excluded due to premature bracing discontinuation. Inclusion criteria were as follows: Risser $0-2$ at the beginning of treatment, curve magnitude $\left(\mathrm{C}_{\mathrm{M}}\right) 20^{\circ}-50^{\circ} \mathrm{Cobb}$, full-time brace prescription and no previous treatment. Curves between $20^{\circ}$ and $25^{\circ} \mathrm{Cobb}$ degrees were included only in the presence of documented progression. The latter was assessed on two consecutive $\mathrm{X}$-rays taken at 6-month interval and was defined as an increase greater than $5^{\circ}$ in $C_{M}$ (Cobb's method) [26]. The minimum duration of follow-up was 24 months after the end of treatment. Compliance to treatment was categorized as: brace worn as prescribed (complete), brace removed for 1 month of a year (incomplete A), brace removed for 2 months of a year (incomplete B), brace removed during school hours (incomplete $\mathrm{C}$ ), and brace worn overnight only (incomplete D).

\section{Bracing protocol}

All patients were prescribed with full-time bracing (i.e., max 22 hours daily, min 18 hours daily). The patients with thoracolumbar and lumbar curves were treated with PASB [27], instead thoracic and double major curves with Lyon or Milwaukee Brace in according to age. In order to maximize treatment adherence, patients were always followed by the same physician. Controls were performed every two months until Risser 3, and every three months, always in the presence of parents. Close checks were also necessary to maximize the efficacy of bracing over time. Frequent checks allowed verifying and implementing compliance by establishing an open and friendly relationship with the patients and their parents.

Weaning was started when ring-apophysis fusion was seen to begin on a latero-lateral (LL) radiograph view [28], which corresponds to a Risser sign 4 or 5 on an antero-posterior (AP) standing radiograph view. Weaning consisted of 2 to 4 hours bracing reduction at 2-month intervals.

Compliance to treatment was established via in-person interviews at each clinical examination. Responses of patients were ratified by their parents and indirectly by the assessment of the hump course [29]. In cases of hump or curve worsening, a thorough evaluation of the patient's behaviour was performed and a stronger parental involvement encouraged.

\section{Endpoints}

For the present study, only the X-ray performed at conventional times were considered: beginning of treatment $\left(t_{1}\right)$, 4-6 months after the beginning of treatment $\left(t_{2}\right)$, intermediate time between $t_{1}$ and $t_{4}\left(t_{3}\right)$, end of weaning $\left(t_{4}\right)$, 2-year minimum follow-up $\left(t_{5}\right)$. For each patient, $\mathrm{AP}$ and LL view standing $\mathrm{X}$-rays of the whole spine were performed. X-rays before treatment $\left(t_{1}\right)$ as well as those at $t_{4}$ and $t_{5}$ were taken while out of brace. All other radiographic controls were performed with the patient wearing the brace, in order to assess its corrective action. The first X-ray was obtained at 4-6 months from the beginning of treatment. All other controls were performed once a year. The AP view was used to determine the patient's skeletal age (Risser's sign) and to obtain the $C_{M}$ and torsion of the apical vertebra ( $\mathrm{T}_{\mathrm{A}}$ : Perdriolle's method). Measurements were obtained by two independent observers. The end-vertebrae were pre-selected to reduce inter-observer error [26]. Curves were classified according to SRS in thoracic, thoracolumbar, lumbar, and double major. As recommended by the SRS Committee on Bracing and Non-operative Management, outcomes were classified as follows: (1) correction (percentage of patients with $\leq 5^{\circ}$ curve progression), (2) stabilization (percentage of patients with $>-5$ and $<5^{\circ}$ changes in $C_{M}$ ), (3) progression (percentage of patients with $\geq 5^{\circ}$ progression at maturity), and (4) percentage of patients with curves exceeding $45^{\circ}$ at maturity and those who were recommended for or had undergone surgery.

\section{Statistical analysis}

Descriptive analyses, independent t-tests and chi-square tests were used to describe and analyze the characteristics of the study population at baseline, as appropriate.

Differences between baseline (t1) and the end of the study (t5) in Cobb and Perdriolle degrees over compliance with treatment was tested using One Way ANOVA adjusted for Bonferroni.

The influence of possible confounders on Cobb and Perdriolle degree was tested with a multiple linear regression model. The model was run separately for juvenile and adolescent scoliosis using the differences between baseline (t1) and the end of the study (t5) in Cobb and Perdriolle degrees as dependent variable and orthopaedic 
corset compliance as independent variable. The model was adjusted for: gender, age at baseline, length of treatment, type of brace and, respectively, Cobb and Perdriolle degree at baseline.

Furthermore, ANOVA for repeated measures with the last observation carried forward (LOCF) was used to examine the within and between group effects on the dependent variable.

The Anova was repeated separately for juvenile and adolescent scoliosis using the Cobb and Perdriolle degree, respectively, as dependent variable. Time from baseline (t1) to 5 and the orthopedic corset compliance was used to define the within and between groups variability. Assumptions of repeated measures were tested; when sphericity assumption was violated, Greenhouse-Geisser adjustments were applied.

Statistical significance was set as a p value $\leq 0.05$ (twotailed). The analyses were performed by using SPSS 13.0 software.

\section{Results}

Sample characteristics according the type of scoliosis are displayed in Table 1. The age at baseline [mean (SD)] was 9.0 (1.2) and 12.6 for Juvenile and adolescent scoliosis, while the length of treatment was 80.3 (26.2) month and 57.2 (18.4), respectively. The results from our study showed that at follow-up the compliance was: brace worn as prescribed (Complete) 61.1\%; brace removed for 1 month of a year (incomplete A) 5.2\%; brace removed for 2 months of a year (incomplete B) 10.7\%; brace removed during school hours (incomplete C) 14.2\%; brace worn overnight only (incomplete D) $8.8 \%$. Curve correction was accomplished in 301/319 of Complete, 19/27 brace

Table 1 Sample characteristics

\begin{tabular}{cccc}
\hline & & JIS & AIS \\
\hline Gender & Male & 116 & 367 \\
Curve & Female & 11 & 28 \\
& Double major & 5 & 11 \\
& Lumbar & 24 & 96 \\
Brace & Thoracic & 34 & 77 \\
& Thoracolumbar & 64 & 211 \\
& Lion & 18 & 138 \\
Compliance & Milw & 52 & 42 \\
& Pasb & 57 & 215 \\
& Complete & 66 & 253 \\
& Incomplete A & 12 & 15 \\
& Incomplete B & 25 & 31 \\
& Incomplete C & 14 & 60 \\
& Incomplete D & 10 & 36 \\
& Total (522) & 127 & 395 \\
\hline
\end{tabular}

removed for 1 month of a year (incomplete A), 25/56 brace removed for 2 months of a year (incomplete B), 52/74 brace removed during school hours (incomplete C), 27/46 brace worn overnight only (incomplete D). Surgery Referral was $3 / 319$ of Complete, $1 / 27$ brace removed for 1 month of a year (incomplete A), 4/56 brace removed for 2 months of a year (incomplete B), 2/74 brace removed during school hours (incomplete C), 4/46 brace worn overnight only (incomplete D).

Cobb mean value was $29.8 \pm 7.5 \mathrm{SD}$ at beginning and $17.1 \pm 10.9$ SD at follow-up. Perdriolle was $13.2 \pm 5.6$ SD at beginning and $7.6 \pm 4.8$ at follow-up. Differences in Cobb and Perdriolle degrees between baseline (t1) and end of study (t5) over compliance with brace treatment are showed in Table 2 and 3, respectively.

The Figure 1 showed the difference between $\mathrm{t} 5$ and $\mathrm{t} 1$ in Cobb and Perdriolle degrees over type of scoliosis and Compliance. Both Cobb and Perdriolle degree amelioration was significantly higher in patients with complete compliance over all other groups, both in juvenile, both in adolescent scoliosis. In adolescent scoliosis, also patients that removed brace for 2 months of a year (incomplete B) showed higher mean differences than those that removed brace during school hours (incomplete $\mathrm{C}$ ).

Univariate analysis at baseline have shown significant differences over adherence groups only between type of brace and Perdriolle degree in patient with adolescent scoliosis (higher use of PASB $(\mathrm{p}=0.02)$ and lower Perdriolle degree $(\mathrm{p}=0.0002)$ in patient with complete compliance). No significant differences were found according to gender and type of curve. Similarly, no differences were found in Cobb degree at baseline over compliance groups.

Multiple regression [Tables 4 and 5] showed that compliance with treatment was significantly associated with greater improvement in Cobb and Perdriolle degrees. Length of treatment was always significantly associated with a lower reduction, while age and Cobb degree at baseline were associated respectively with a lower and higher Cobb degrees reduction only in adolescent scoliosis. PASB brace was significantly associated with a higher Cobb and Perdriolle reduction in adolescent scoliosis.

\section{Juvenile}

The evaluation of Cobb degrees for each level of compliance during each time point in the juvenile curves showed the following results [Figure 2a]. The assumption of sphericity was not met, so all comparisons were made using the Greenhouse-Geisser correction. There was a significant main effect for time $(p=0.000)$, for compliance $(p=0.003)$ and for the Interaction $(\mathrm{p}=0.000)$. All time showed within-group differences in the COBB degree, except time 2 versus time 3. All groups showed differences in Cobb degree, but they were significative only between compliance Complete and Incomplete B (brace removed for 
Table 2 COBB degrees distribution according the treatment adherence

\begin{tabular}{|c|c|c|c|c|c|}
\hline \multicolumn{6}{|c|}{ Juvenile scoliosis (JIS) } \\
\hline Compliance (N) & & Baseline & t5 & Difference between $\mathrm{t} 5$ and baseline & Difference over compliance \\
\hline \multirow[t]{2}{*}{ Complete } & mean & 29.4 & 10.9 & -18.5 & Reference \\
\hline & SD & 6.7 & 9.5 & $(p<0.001)$ & \\
\hline \multirow[t]{2}{*}{ Incomplete A } & mean & 29.2 & 21.8 & -7.4 & 11.1 \\
\hline & SD & 6.9 & 9.1 & $(P=0.006)$ & $(p<0.001)$ \\
\hline \multirow[t]{2}{*}{ Incomplete B } & mean & 29.2 & 24.9 & -4.1 & 14.4 \\
\hline & SD & 6.8 & 10.9 & $(p=0.045)$ & $(p<0.001)$ \\
\hline \multirow[t]{2}{*}{ Incomplete C } & mean & 28.8 & 18.4 & -10.4 & 8.1 \\
\hline & SD & 8.1 & 5.5 & $(p<0.001)$ & $(p<0.001)$ \\
\hline \multirow[t]{2}{*}{ Incomplete D } & mean & 32.6 & 24.2 & -8.4 & 10.1 \\
\hline & SD & 7.5 & 6.2 & $(p<0.001)$ & $(p<0.001)$ \\
\hline \multirow[t]{2}{*}{ Total } & mean & 29.5 & 16.5 & -13 & \\
\hline & SD & 6.9 & 11.0 & $(p<0.001)$ & \\
\hline \multicolumn{6}{|c|}{ Adolescent scoliosis (AIS) } \\
\hline \multicolumn{2}{|l|}{ Compliance (N) } & Baseline & t5 & Difference between $\mathrm{t} 5$ and baseline & Difference over compliance \\
\hline \multirow[t]{2}{*}{ Complete } & mean & 30.5 & 13.1 & -17.4 & Reference \\
\hline & SD & 8.0 & 10.0 & $(p<0.001)$ & \\
\hline \multirow[t]{2}{*}{ Incomplete A } & mean & 30.5 & 20.8 & -9.7 & 7.7 \\
\hline & SD & 5.1 & 6.8 & $(p<0.001)$ & $(p<0.001)$ \\
\hline \multirow[t]{2}{*}{ Incomplete B } & mean & 28.6 & 24.2 & -4.4 & 13 \\
\hline & SD & 7.0 & 8.0 & $(p=n s)$ & $(p<0.001)$ \\
\hline \multirow[t]{2}{*}{ Incomplete C } & mean & 30.2 & 20.1 & -10.1 & 7.3 \\
\hline & SD & 6.7 & 7.5 & $(p<0.001)$ & $(p<0.001)$ \\
\hline \multirow[t]{2}{*}{ Incomplete D } & mean & 31.5 & 23.1 & -8.4 & 9 \\
\hline & SD & 8.8 & 9.0 & $(p<0.001)$ & $(p<0.001)$ \\
\hline \multirow[t]{2}{*}{ Total } & mean & 30.4 & 16.3 & -14.1 & \\
\hline & SD & 7.7 & 10.2 & $(p<0.001)$ & \\
\hline
\end{tabular}

2 months of a year $)(-9.268 ; \mathrm{P}=0.000)$ and Complete and Incomplete D (brace worn overnight only) ( -9.444 ; $\mathrm{p}=0.013)$. Significant interactions showed that the effect of time was different for the five compliance groups: as it is reasonable, the maximum compliance had greater Cobb degree reduction than the other groups during time, especially in late treatment. There was significant greater improvement in COBB degree favouring "complete compliance" group respect those that worn brace overnight only (incomplete D) at many time points: $\mathrm{t} 3, \mathrm{t} 4$ and $\mathrm{t} 5$ $(\mathrm{P}<0.01)$. Patients with brace removed for 1 month of a year (incomplete A), showed a greater improvement than those that worn brace overnight only (incomplete D) at $\mathrm{t} 3$, without reach the statistical significance $(P=0.052)$.

While the Perdriolle showed these results [Figure 3a]. The assumption of sphericity was not met, so all comparisons were made using the Greenhouse-Geisser correction. There was a significant main effect for time, compliance and Interaction $(\mathrm{p}=0.000)$. There was within-group differences in the COBB degree for all time, except $t 2$ versus $\mathrm{t} 4$ and $\mathrm{t} 5$ and $\mathrm{t} 3$ versus $\mathrm{t} 4$. All groups showed a reduction in Cobb degree, but there was a significant difference only between compliance Complete and Incomplete B (brace removed for 2 months of a year) ( -4.658 ; $\mathrm{P}=0.004$ ) and Incomplete D (brace worn overnight only) (-6.582; $\mathrm{P}=0.005)$. Significant interactions showed that the effect of time was different for the compliance groups: as it is shown in Figure 3, the higher interaction was between compliance complete and incomplete $C$ (brace removed during school hours). There was significant greater improvement in $\mathrm{COBB}$ degree favouring different compliance group versus Compliance D at many time points: Complete compliance in all time $[\mathrm{t} 1(\mathrm{P}=0,018) ; \mathrm{t} 2$ $(\mathrm{P}=0.009) ; \mathrm{t} 3 \quad(\mathrm{P}=0.002), \mathrm{t} 4 \quad(\mathrm{P}=0.000), \mathrm{t} 5 \quad(\mathrm{P}=0.000)]$; Incomplete $\mathrm{A}$ in $\mathrm{t} 2, \mathrm{t} 3, \mathrm{t} 4$ and $\mathrm{t} 5$; Incomplete $\mathrm{C}$ in $\mathrm{t} 1(\mathrm{P}=$ 0.033), t2 ( $\mathrm{P}=0.049), \mathrm{t} 4(\mathrm{P}=0.022)$ and $\mathrm{t} 5(\mathrm{P}=0.047)$. 
Table 3 Perdriolle degrees distribution according the treatment adherence

\begin{tabular}{|c|c|c|c|c|c|}
\hline \multicolumn{6}{|c|}{ Juvenile scoliosis (JIS) } \\
\hline Compliance $(\mathrm{N})$ & & Baseline & t5 & Difference between $\mathrm{t} 5$ and baseline & Difference over compliance \\
\hline \multirow[t]{2}{*}{ Complete } & mean & 12.2 & 6.0 & -6.2 & Reference \\
\hline & SD & 4.1 & 5.6 & $(p<0.001)$ & \\
\hline \multirow[t]{2}{*}{ Incomplete A } & mean & 13.0 & 10.3 & -2.7 & 3.5 \\
\hline & SD & 5.0 & 5.7 & $(p=0.049)$ & $(p=0.013)$ \\
\hline \multirow[t]{2}{*}{ Incomplete B } & mean & 14.1 & 12.5 & -1.6 & 4.6 \\
\hline & SD & 4.7 & 6.1 & $(p=0.199)$ & $(p<0.001)$ \\
\hline \multirow[t]{2}{*}{ Incomplete C } & mean & 10.2 & 8.5 & -1.7 & 4.5 \\
\hline & SD & 2.8 & 2.3 & $(p=0.143)$ & $(p=0.001)$ \\
\hline \multirow[t]{2}{*}{ Incomplete D } & mean & 14.6 & 14.3 & -0.3 & 5.9 \\
\hline & SD & 5.4 & 5.2 & $(p=0.766)$ & $(p<0.001)$ \\
\hline \multirow[t]{2}{*}{ Total } & mean & 12.9 & 8.7 & -4.2 & \\
\hline & SD & 4.9 & 6.2 & $(p<0.001)$ & \\
\hline \multicolumn{6}{|c|}{ Adolescent scoliosis (AIS) } \\
\hline Compliance $(\mathrm{N})$ & & Baseline & t5 & Difference between $\mathrm{t} 5$ and baseline & Difference over compliance \\
\hline \multirow[t]{2}{*}{ Complete } & mean & 12.0 & 6.2 & -5.8 & Reference \\
\hline & SD & 4.1 & 5.2 & $(p<0.001)$ & \\
\hline \multirow[t]{2}{*}{ Incomplete A } & mean & 14.9 & 11.2 & -3.7 & 2.1 \\
\hline & SD & 4.7 & 6.6 & $(p<0.001)$ & $(p<0.001)$ \\
\hline \multirow[t]{2}{*}{ Incomplete B } & mean & 12.9 & 11.4 & -1.5 & 4.3 \\
\hline & SD & 4.1 & 5.6 & $(p<0.09)$ & $(p<0.001)$ \\
\hline \multirow[t]{2}{*}{ Incomplete C } & mean & 14.4 & 10.9 & -3.5 & 2.3 \\
\hline & SD & 4.9 & 5.9 & $(p<0.001)$ & $(p<0.001)$ \\
\hline \multirow[t]{2}{*}{ Incomplete D } & mean & 13.7 & 10.9 & -2.8 & 3.0 \\
\hline & SD & 5.9 & 20.0 & $(p<0.001)$ & $(p<0.001)$ \\
\hline \multirow[t]{2}{*}{ Total } & mean & 12.8 & 8.3 & -4.5 & \\
\hline & SD & 4.6 & 8.3 & $(p<0.001)$ & \\
\hline
\end{tabular}

\section{Adolescent}

The evaluation of Cobb degrees for each level of compliance during each time point in the adolescent curves showed the following results [Figure $2 \mathrm{~b}$ ]. The assumption of sphericity was not met, so all comparisons were made using the Greenhouse-Geisser correction. There was a significant main effect for time, for compliance and for the Interaction $(p=0.000)$. There was within-group differences in the COBB degree for all time, except $t 2$ versus $t 4$ and $t 3$ versus $t 4$. All groups showed a reduction in Cobb degree, but the group with complete compliance had significant differences with all the other except of subgroup that removed brace for 1 month of a year (incomplete A), $(-4.070 ; \mathrm{P}=0.481)$. Significant interactions showed that the effect of time was different for the five compliance groups: as it is reasonable, the maximum compliance had greater Cobb degree reduction than the other groups during time, especially in late treatment. There was significant greater improvement in COBB degree favouring "complete compliance" group respect Incomplete D (brace worn overnight only) at many time points: $\mathrm{t} 3, \mathrm{t} 4$ and $\mathrm{t} 5(\mathrm{P}<$ 0.01). Furthermore the group that removed brace for 1 month of a year (incomplete A) showed a greater improvement than those that worn brace overnight only (incomplete D) at $\mathrm{t} 2$ and $\mathrm{t} 4$, without reach the statistical significance $(\mathrm{P}=0.058$ and 0.055 respectively); subgroup that removed brace during school hours (incomplete $\mathrm{C}$ ) showed a greater improvement at $\mathrm{t} 4(\mathrm{P}=0.043)$.

While the Perdriolle showed these results [Figure 3b]. The assumption of sphericity was not met, so all comparisons were made using the Greenhouse-Geisser correction. There was a significant main effect for time, compliance and Interaction $(\mathrm{p}=0.000)$. There was withingroup differences in the Cobb degree for all time, except t2 vs t5. All groups showed a reduction in Perdriolle degree, but the group with complete compliance had significant differences with all the other except that "brace removed for 1 month of a year (incomplete A)" 


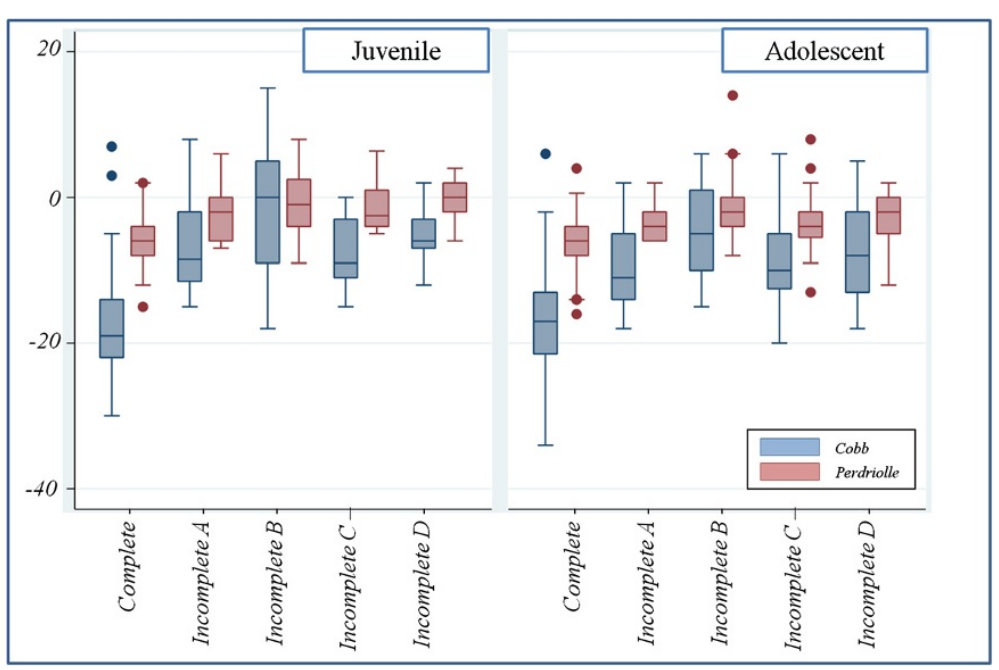

Figure 1 Difference between $\mathrm{t} 5$ and $\mathrm{t} 1$ in Cobb degree (blue boxes) and Perdriolle degree (red boxes) over type of scoliosis and Compliance ( 0 = complete; 1 = brace removed for 1 month of a year (incomplete A); $2=$ brace removed for 2 months of a year (incomplete B); $3=$ brace removed during school hours (incomplete C); 4 = Incomplete D).

compliance $(-3.090 ; \mathrm{P}=0.10)$. Significant interactions showed that the effect of time was different for the five compliance groups: as it is reasonable, the maximum compliance had greater Perdriolle degree reduction than the other groups during time, especially in late treatment. There was significant greater improvement in COBB degree favouring "complete compliance" group respect worn brace overnight only (incomplete $\mathrm{D}$ ) at many time points: $\mathrm{t} 1(\mathrm{P}=0.035) \mathrm{t} 3(\mathrm{P}=0.002), \mathrm{t} 4 \quad(\mathrm{P}=0.000)$ and t5 $(\mathrm{P}=0.001)$

\section{Intention to treat analysis}

A total of 642 patients were eligible for inclusion criteria. Of these, 522 patients have a definite outcome and 14 of these have indication to surgery, while 120 have abandon the treatment. 65 of 120 patients who abandoned the treatment were revaluate at follow up and only 9 of these have indication to surgery (Table 6).

The rate of surgery referral was $2.1 \%$ among patients with definite outcome and $12.1 \%$ among those with drop-out.

\section{Discussion}

The aim of the present study was to evaluate the relationship between compliance to bracing wear and the progression of scoliotic curves in patients with idiopathic adolescent (AIS) or juvenile scoliosis (JIS) treated with PASB, Lyon or Milwaukee brace. Our results indicate that

Table 4 Multiple linear regression: results for Cobb degree difference (t5 vs baseline)

\begin{tabular}{|c|c|c|c|c|c|c|c|}
\hline \multirow{2}{*}{\multicolumn{2}{|c|}{$\begin{array}{l}\text { Cobb degree difference } \\
\text { Independent variables }\end{array}$}} & \multicolumn{3}{|c|}{ JIS (R2 = 0.55) } & \multicolumn{3}{|c|}{ AIS (R2 = 0.48) } \\
\hline & & \multirow{2}{*}{$\frac{\text { B coefficient }}{-}$} & \multirow{2}{*}{$\begin{array}{c}\text { Standard error } \\
-\end{array}$} & \multirow{2}{*}{$\begin{array}{l}\mathbf{p} \\
-\end{array}$} & \multirow{2}{*}{$\frac{\text { B coefficient }}{-}$} & \multirow{2}{*}{$\begin{array}{c}\text { Standard error } \\
-\end{array}$} & \multirow{2}{*}{$\begin{array}{l}\mathrm{p} \\
-\end{array}$} \\
\hline Adherence & Complete (reference) & & & & & & \\
\hline & Incomplete A & 8.57 & 2.36 & 0.000 & 6.54 & 1.49 & 0.000 \\
\hline & Incomplete B & 13.77 & 1.78 & 0.000 & 11.39 & 1.08 & 0.000 \\
\hline & Incomplete C & 7.30 & 2.43 & 0.003 & 6.88 & 0.83 & 0.000 \\
\hline & Incomplete D & 10.35 & 2.53 & 0.000 & 8.60 & 1.02 & 0.000 \\
\hline \multicolumn{2}{|c|}{ Gender } & 4.02 & 2.53 & 0.114 & 0.85 & 1.11 & 0.444 \\
\hline \multicolumn{2}{|c|}{ Age baseline } & 0.99 & 0.61 & 0.109 & 0.87 & 0.25 & 0.001 \\
\hline \multicolumn{2}{|c|}{ Length of treatment } & 0.08 & 0.03 & 0.008 & 0.11 & 0.02 & 0.000 \\
\hline \multicolumn{2}{|c|}{ Cobb at baseline } & -0.11 & 0.11 & 0.321 & -0.14 & 0.04 & 0.001 \\
\hline \multirow[t]{3}{*}{ Type of brace } & Lyon (Reference) & - & - & - & - & - & - \\
\hline & Milwaukee & 0.76 & 2.14 & 0.724 & -0.29 & 1.01 & 0.776 \\
\hline & Pasb & -1.76 & 2.21 & 0.428 & -2.61 & 0.65 & 0.000 \\
\hline
\end{tabular}


Table 5 Multiple linear regression: results for Perdriolle degree difference (t 5 vs baseline)

\begin{tabular}{|c|c|c|c|c|c|c|}
\hline \multirow{2}{*}{$\begin{array}{c}\text { Perdriolle degree difference } \\
\text { Independent variables }\end{array}$} & \multicolumn{3}{|c|}{ JIS (R2 = 0.40) } & \multicolumn{3}{|c|}{ AIS $(R 2=0.29)$} \\
\hline & B coefficient & Standard error & $p$ & B coefficient & Standard error & $p$ \\
\hline \multirow{5}{*}{ Adherence } & Complete (reference) & - & - & - & - & - \\
\hline & Incomplete A & 1.92 & 1.19 & 0.109 & 1.35 & 0.78 \\
\hline & Incomplete B & 4.23 & 0.89 & 0.000 & 3.97 & 0.56 \\
\hline & Incomplete C & 3.35 & 1.22 & 0.007 & 1.94 & 0.44 \\
\hline & Incomplete D & 4.65 & 1.27 & 0.000 & 2.46 & 0.54 \\
\hline \multicolumn{2}{|c|}{ Gender } & 2.15 & 1.29 & 0.099 & 0.18 & 0.58 \\
\hline \multicolumn{2}{|c|}{ Age baseline } & 0.18 & 0.31 & 0.558 & 0.09 & 0.13 \\
\hline \multicolumn{2}{|c|}{ Length of treatment } & 0.05 & 0.01 & 0.001 & 0.05 & 0.01 \\
\hline \multicolumn{2}{|c|}{ Perdriolle at baseline } & -0.04 & 0.08 & 0.633 & -0.02 & 0.04 \\
\hline & Lyon (Reference) & - & - & - & - & - \\
\hline \multirow[t]{2}{*}{ Type of brace } & Milwaukee & -0.18 & 1.08 & 0.865 & 0.21 & 0.52 \\
\hline & Pasb & -0.47 & 1.09 & 0.669 & -0.93 & 0.34 \\
\hline
\end{tabular}

the use of brace as prescribed may alter the natural history of AIS and JIS. In particular, patients with complete compliance o that removed brace for 1 month of a year (incomplete A) show more favourable outcomes than those that removed brace during school hours (incomplete C) or worn brace overnight only (incomplete D). The type of brace influences the compliance, such that adherence to treatment is higher with PASB than Lyon or Milwaukee brace. Interestingly, AIS patients show a better compliance to bracing than those with JIS. Finally, PASB provides better correction both in adolescent and juvenile curves.

Recent studies have assessed compliance to bracing by either questionnaires [15-18] or a sensor attached to the brace. Notably, compliance measured by sensors was lower compared with that reported by patients [13,22]. In the present investigation, sensors could not be used as they were not available at the time of study beginning.
However, the evaluation of compliance was performed by a single surgeon and correlated with clinical signs and information obtained by the patient parents.

Overall, a complete compliance was recorded in $61 \%$ of the study sample (51\% for juvenile and $64 \%$ for adolescent), which is in agreement with the adherence rate reported in the literature (33-97\%, mean: 69\%). [10,19-25,30,31] Donzelli et al. evaluated the compliance in 68 patients for about five months with an electronic monitor and recorded $91.7 \%$ of full compliance. Patients with full-time brace prescription showed a higher adherence than those with part-time bracing. Interestingly, higher compliance rates were observed in patients fulfilling the SOSORT criteria [13].

Brox et al. evaluated compliance and efficacy of bracing without electronic monitor in 495 patients an 389 (79\%) patients were registered as compliant, 106 as non-compliant, the progression of curve at long-term
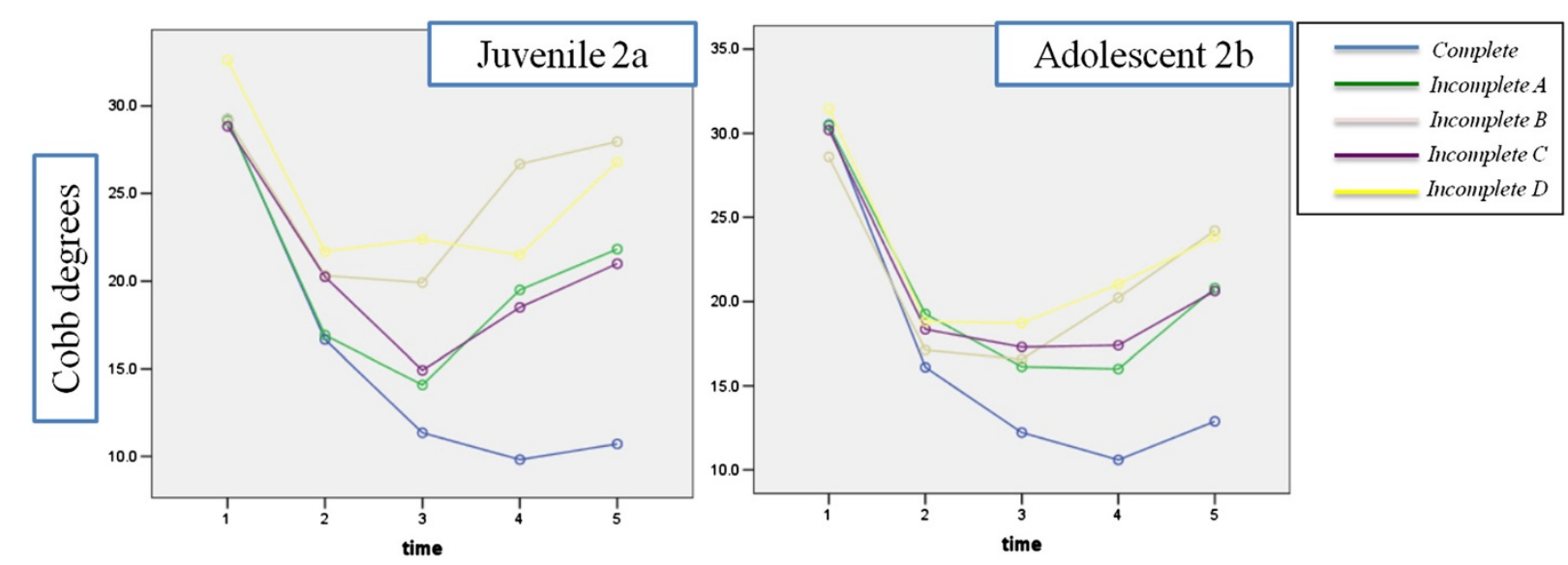

Figure 2 Changes in Cobb degrees for each level of compliance during each time point. 

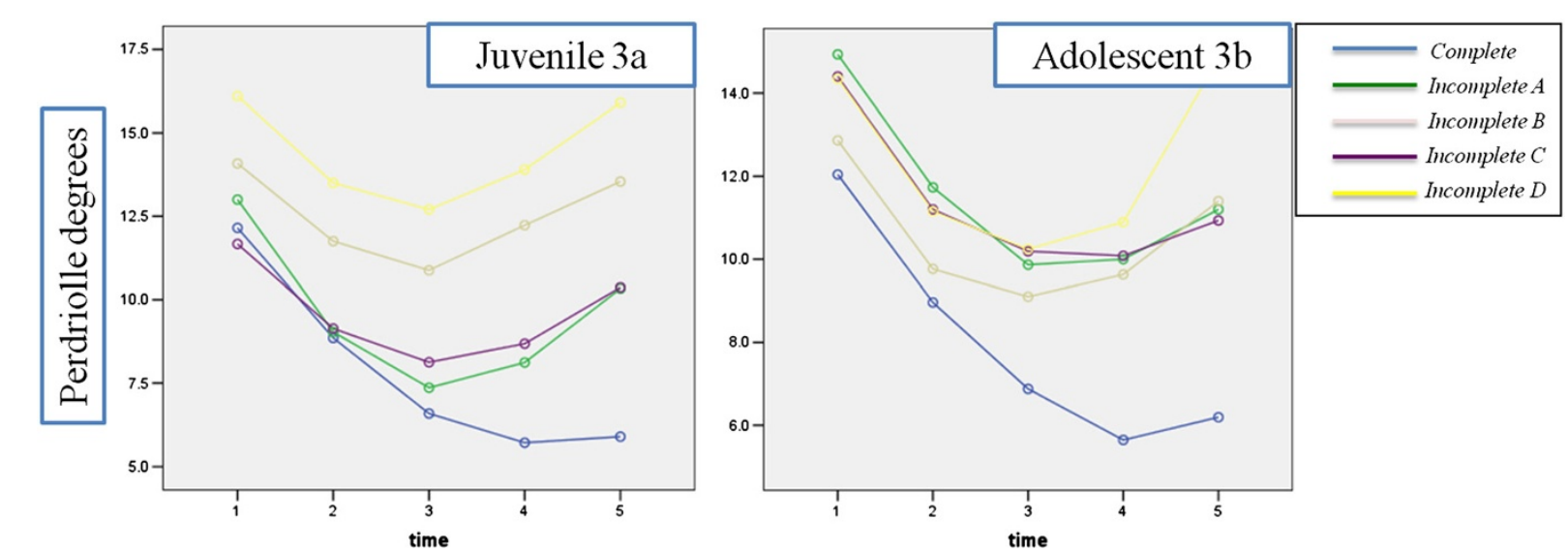

Figure 3 Changes in Perdriolle degrees for each level of compliance during each time point.

were $24 \%$ in compliant and $65 \%$ in non-compliant, the surgical rate was $3.5 \%$ versus $24 \%$ [32]. Rahman et al. showed similar results using an electronic monitor to evaluate time bracing in 34 patients and reported curve progression in $11 \%$ with high compliance and $56 \%$ with low compliance [21].

It is noteworthy that, different from most studies, in the present investigation compliance was monitored for the whole duration of treatment. A more in-depth analysis of our data shows the compliance is usually higher at the beginning of treatment and that the months of non compliance were often in the summer. The greater correction was obtained during the first six months in all the study sample, after which patients with complete compliance continue to improve, while those categorized as incomplete B (brace removed for 2 months of a year) and $\mathrm{D}$ show a negative trend, which however did not affect the final outcome. This pattern can be explained by the motivation of the patient is initially higher and gradually decreases during the course of treatment.
These considerations may also explain the satisfactory results and the low rate of surgery $(2.4 \%$ in group that removed brace during school hours (incomplete C), 5.1\% in group that worn brace overnight only (incomplete D) and $0 \%$ in the other groups).

The point of strengths of this study are the large study sample, the long term follow-up, the radiological evaluation, the different type of braces studied and the management of patients according to the SOSORT guidelines.

\section{Conclusion}

Use the brace as prescribed may alter the natural history of AIS and JIS and curve progression and referral to surgery are lower in patients with high brace compliance. Bracing discontinuation up to 1 month a year does not impact on the treatment outcome. Conversely, wearing the brace only overnight and bracing discontinuation up to 2 months a year is associated with a high rate of curve progression.

Table 6 Sample of patients that abandon the treatment

\begin{tabular}{lcc}
\hline & Abandon without F-up no./total no. (\%) & Abandon with F-up no./total no. (\%) \\
\hline Risser 0 & $1 / 55(1,9 \%)$ & $2 / 65(1,5 \%)$ \\
Risser 1 & $3 / 55(5,4 \%)$ & $1 / 65(3 \%)$ \\
Risser 2 & $12 / 55(21,8 \%)$ & $8 / 65(12,5 \%)$ \\
Risser 3 & $37 / 55(67,2 \%)$ & $40 / 65(61,5 \%)$ \\
Risser 4 & $2 / 55(3,6 \%)$ & $14 / 65(21,5 \%)$ \\
Curve correction at time of abandon & $38 / 55(69,1 \%)$ & $55 / 65(84,6 \%)$ \\
Curve stabilization at time of abandon & $15 / 55(27,3 \%)$ & $4 / 65(6,1 \%)$ \\
Curve progression at time of abandon & $2 / 55(3,6 \%)$ & $6 / 65(9,3 \%)$ \\
Curve correction at follow-up & - & $19 / 65(29,2 \%)$ \\
Curve stabilization at follow-up & - & $30 / 65(46,2 \%)$ \\
Curve progression at follow-up & - & $16 / 65(24,6 \%)$ \\
Indication to surgery & $55 / 55(100 \%)$ & $9 / 65(13,8 \%)$
\end{tabular}




\section{Abbreviations}

JIS: Juvenile idiopathic scoliosis; AIS: Adolescent idiopathic scoliosis; SRS: Scoliosis research society; SOSORT: Society on scoliosis orthopedic and rehabilitation treatment; PASB: Progressive action short brace; CM: Curve magnitude; LL: Latero-lateral; AP: Antero-posterior; $\mathrm{T}_{\mathrm{A}}$ : Torsion of the apical vertebra.

\section{Competing interests}

The authors declare that they have no competing interests.

\section{Authors' contributions}

AGA participated in the conception, design and coordination, and to acquisition of data, analysis and interpretation of data and drafted the manuscript. MG, FF and VG helped to draft the manuscript. AP participated in the design of the study and performed the statistical analysis. EM participated in the design of the study and helped to draft the manuscript. All authors read and approved the final manuscript.

\section{Acknowledgements}

We thank Prof. Lorenzo Aulisa who made a substantial contributions to conception, design, acquisition and interpretation of data.

\section{Author details}

'U.O.C. of Orthopedics and Traumatology, Children's Hospital Bambino Gesù, Institute of Scientific Research, P.zza S. Onofrio, Rome 4-00165, Italy.

2Department of Orthopedics, University Hospital "Agostino Gemelli", Catholic University of the Sacred Heart School of Medicine, Rome 00168, Italy. ${ }^{3}$ Institute of public health, University Hospital "Agostino Gemelli", Catholic University of the Sacred Heart School of Medicine, Rome 00168, Italy.

${ }^{4}$ University of Cassino, Cassino, FR 03043, Italy.

Received: 22 May 2014 Accepted: 6 June 2014

Published: 13 June 2014

\section{References}

1. Parent S, Newton PO, Wenger DR: Adolescent idiopathic scoliosis: etiology, anatomy, natural history, and bracing. Instr Course Lect 2005, 54:529-536.

2. Weinstein SL, Dolan LA: BrAIST: the bracing in adolescentidiopathic scoliosis trial. US National Institute of Health; 2007. http://www.clinicaltrials.gov/ct/ show/NCT00448448.

3. Dolan LA, Wright JG, Weinstein SL: Effects of bracing in adolescents with idiopathic scoliosis. N Engl J Med 2014, 370(7):681. Feb 13.

4. Aulisa AG, Guzzanti V, Galli M, Perisano C, Falciglia F, Aulisa L: Treatment of thoraco-lumbar curves in adolescent females affected by idiopathic scoliosis with a progressive action short brace (PASB): assessment of results according to the SRS committee on bracing and nonoperative management standardization criteria. Scoliosis 2009, 4:21.

5. Aulisa AG, Guzzanti V, Perisano C, Marzetti E, Specchia A, Galli M, Giordano $M$, Aulisa L: Determination of quality of life in adolescents with idiopathic scoliosis subjected to conservative treatment. Scoliosis 2010, 5:21.

6. Negrini S, Minozzi S, Bettany-Saltikov J, Zaina F, Chockalingam N, Grivas TB, Kotwicki T, Maruyama T, Romano M, Vasiliadis ES: Braces for idiopathic scoliosis in adolescents. Spine (Phila Pa 1976) 2010, 35:1285-1293.

7. Aulisa AG, Guzzanti V, Marzetti E, Giordano M, Falciglia F, Aulisa L: Brace treatment in juvenile idiopathic scoliosis: a prospective study in accordance with the SRS criteria for bracing studies - SOSORT award 2013 winner. Scoliosis 2014, 9:3. Apr 23.

8. Aulisa AG, Guzzanti V, Perisano C, Marzetti E, Falciglia F, Aulisa L: Treatment of lumbar curves in scoliotic adolescent females with progressive action short brace: a case series based on the Scoliosis Research Society Committee Criteria. Spine (Phila Pa 1976) 2012, 37(13):E786-E791. Jun 1.

9. Negrini S, Atanasio S, Fusco C, Zaina F: Effectiveness of complete conservative treatment for adolescent idiopathic scoliosis (bracing and exercises) based on SOSORT management criteria: results according to the SRS criteria for bracing studies - SOSORT Award 2009 Winner. Scoliosis 2009, 4:19.

10. Rahman T, Bowen JR, Takemitsu M, Scott C: The association between brace compliance and outcome for patients with idiopathic scoliosis. J Pediatr Orthop 2005, 25:420-422.

11. Seifert J, Selle A, Flieger C, Gunther KP: Compliance as a prognostic factor in the treatment of idiopathic scoliosis. Orthopade 2009, 38:151-158.
12. Rowe DE, Bernstein SM, Riddick MF, Adler F, Emans JB, Gardner-Bonneau D: A meta-analysis of the efficacy of non-operative treatment for idiopathic scoliosis. J Bone Joint Surg Am 1997, 79:664-672

13. Donzelli S, Zaina F, Negrini S: In defense of adolescents: They really do use braces for the hours prescribed, if good help is provided. Results from a prospective everyday clinic cohort using thermobrace. Scoliosis 2012, 7(1):12. May 31.

14. Negrini S, Aulisa AG, Aulisa L, Circo AB, de Mauroy JC, Durmala J, Grivas TB, Knott P, Kotwicki T, Maruyama T, Minozzi S, O'Brien JP, Papadopoulos D, Rigo M, Rivard CH, Romano M, Wynne JH, Villagrasa M, Weiss HR, Zaina F: 2011 SOSORT Guidelines: Orthopaedic and Rehabilitation Treatment of Idiopathic Scoliosis During Growth. Scoliosis 2011, 7(1):3

15. Noonan K, Weinstein SL, Jacobson WC, Dolan LA: Use of the Milwaukee brace for progressive idiopathic scoliosis. J Bone Joint Surg Am 1996, 78:557-567.

16. Lonstein JE, Winter RB: The Milwaukee brace for the treatment of adolescent idiopathic scoliosis. J Bone Joint Surg Am 1994, 76:1207-1221.

17. Wiley JW, Thomson JD, Mitchell TM, Mitchell TM, Smith BG, Banta JV: Effectiveness of the Boston brace in treatment of large curves in adolescent idiopathic scoliosis. Spine 2000, 25:2326-2332.

18. DiRaimondo CV, Green NE: Brace-wear compliance in patients with adolescent idiopathic scoliosis. J Pediatr Orthop 1988, 8:143-146.

19. Vandal S, Rivard CH, Bradet R: Measuring the compliance behavior of adolescents wearing orthopedic braces. Issues Compr Pediatr Nurs 1999, 22(2-3):59-73.

20. Havey R, Gavin T, Patwardhan A, Pawelczak S, Ibrahim K, Andersson GB, Lavender S: A reliable and accurate method for measuring orthosis wearing time. Spine (Phila Pa 1976) 2002, 27(2):211-214.

21. Rahman T, Borkhuu B, Littleton AG, Sample W, Moran E, Campbell S, Rogers $\mathrm{K}$, Bowen JR: Electronic monitoring of scoliosis brace wear compliance. J Child Orthop 2010, 4(4):343-347.

22. Takemitsu M, Bowen JR, Rahman T, Glutting JJ, Scott CB: Compliance monitoring of brace treatment for patients with idiopathic scoliosis. Spine 2004, 29(18):2070-2074. discussion 2074.

23. Helfenstein A, Lankes M, Ohlert K, Varoga D, Hahne HJ, Ulrich HW Hassenpflug J: The objective determination of compliance in treatment of adolescent idiopathic scoliosis with spinal orthoses. Spine (Phila Pa 1976) 2006, 31(3):339-344.

24. Nicholson GP, Ferguson-Pell MW, Smith K, Edgar M, Morley T: Quantitative measurement of spinal brace use and compliance in the treatment of adolescent idiopathic scoliosis. Stud Health Technol Inform 2002, 91:372-377.

25. Katz DE, Herring JA, Browne RH, Kelly DM, Birch JG: Brace wear control of curve progression in adolescent idiopathic scoliosis. J Bone Joint Surg Am 2010, 92(6):1343-1352.

26. Morrissy RT, Goldsmith GS, Hall EC, Kehl D, Cowie GH: Measurement of the Cobb angle on radiographs of patients who have scoliosis. Evaluation of intrinsic error. J Bone Joint Surg Am 1990, 72(3):320-327.

27. Aulisa AG, Mastantuoni G, Laineri M, Falciglia F, Giordano M, Marzetti E, Guzzanti V: Brace technology thematic series: the progressive action short brace (PASB). Scoliosis 2012, 7:6. Feb 23.

28. Bick EM, Copel JW: The ring apophysis of the human vertebra; contribution to human osteogeny. J Bone Joint Surg Am 1951, 33-A(3):783-787.

29. Aulisa AG, Guzzanti V, Perisano C, Marzetti E, Menghi A, Giordano M, Aulisa $\mathrm{L}$ : Correlation between hump dimensions and curve severity in idiopathic scoliosis before and after conservative treatment. Spine (Phila Pa 1976) 2011. Jan 8 [Epub ahead of print].

30. Morton A, Riddle R, Buchanan R, Katz D, Birch J: Accuracy in the prediction and estimation of adherence to bracewear before and during treatment of adolescent idiopathic scoliosis. J Pediatr Orthop 2008, 28(3):336-341.

31. Muller C, Fuchs K, Winter C, Rosenbaum D, Schmidt C, Bullmann V, Schulte $T L$ : Prospective evaluation of physical activity in patients with idiopathic scoliosis or kyphosis receiving brace treatment. Eur Spine J 2011, 20(7):1127-1136.

32. Brox Jl, Lange JE, Gunderson RB, Steen H: Good brace compliance reduced curve progression and surgical rates in patients with idiopathic scoliosis. Eur Spine J 2012, 21(10):1957-1963.

doi:10.1186/1748-7161-9-6

Cite this article as: Aulisa et al.: Correlation between compliance and brace treatment in juvenile and adolescent idiopathic scoliosis: SOSORT 2014 award winner. Scoliosis 2014 9:6. 